Theoretical Physics, Astrophysics and Cosmology

Vol. 2, No 2, p. 9 - 21, v1, 30 November 2007

Online: TPAC: 2525-007 v2, 28 September 2012
ISSN 1819-9135; ISSN 1991-3919 (e)

(C) 2007, 2012 CTPA. All rights reserved. DOI: $10.9751 /$ TPAC.2525-007

\title{
Quantization of complex harmonic oscillator and the zero-point energy vanishing effect
}

\author{
Zahid Zakir $^{1}$
}

\begin{abstract}
The system of two harmonic oscillators with different sign frequencies is presented as a positive-frequency oscillator with a complex generalized coordinate where there are a global $\mathrm{U}(1)$ symmetry and a charge conjugation symmetry (Csymmetry). It is shown that two pairs of ladder operators, appearing at the frequency decomposition of canonical variables, are not mutually charge-conjugate and their standard interpretation as operators of charge-conjugate quanta breaks the Csymmetry. Bilinear operator identities for the ladder operators are found allowed the theory to obey the C-symmetry restrictions and to express observables through the mutually charge-conjugate operators. These identities hold for C-symmetric interactions also. It is shown that C-symmetry eliminates a ground state zero-point charge and a zero-point energy. The uncertainty relations are generalized for nonhermitian canonical variables and it is shown that the charge-conjugation symmetric ground states do not quantized. It is shown that the C-symmetric interactions do not contribute to the ground state energy in all orders of perturbation theory.
\end{abstract}

PACS: 03.65.Ge, 11.30.Er, 1130.Ly, 11.90. $+t$

Key words: Hamiltonian dynamics, discrete symmetries, quantization, oscillator, uncertainty relations

\section{Content}

Introduction

1. Canonical formalism for the complex harmonic oscillator ................................................. 11

2. Symmetries of a complex oscillator and their consequences.............................................. 13

3. New uncertainty relations for complex canonical variables and a ground state energy .......16

4. Conservation of the operator identities for C-symmetric interactions ..................................17

5. A ground state energy at perturbation by the gauge field .................................................... 19

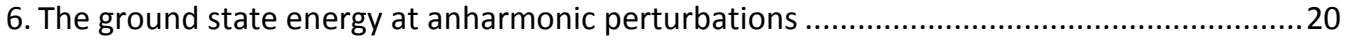

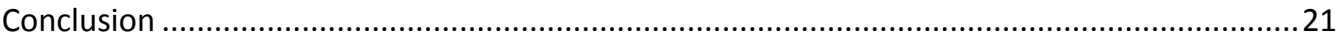

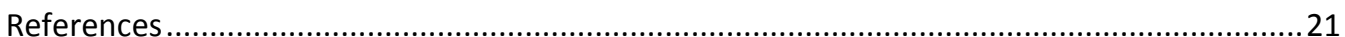

\section{Introduction}

Quantization of systems of harmonic oscillators with various symmetries always drew attention in connection with a problem of a zero-point energy of vacuum and because of unusual effects of the symmetries. Thus, modifications of procedures of quantization of the systems of harmonic oscillators were not restricted to the frameworks of nonrelativistic quantum mechanics and influenced on the development of many applied and fundamental physical theories.

Such exclusive status of this problem is related by that the concept of quantization of many systems in fact has been reduced to representation of their normal modes as sets of harmonic oscillators. On the direct application of the nonrelativistic quantum oscillator model are based not only the standard quantum

${ }^{1}$ Centre for Theoretical Physics and Astrophyics, Tashkent, Uzbekistan; zahidzakir@theorphys.org 
theory of relativistic fields, but also the approaches trying to describe phenomena at the Planck distances. Difficulties or internal inconsistencies in these widely accepted approaches caused doubt that the standard quantization rules of the nonrelativistic systems have not enough adequately adapted to the relativistic systems having also negative-frequency modes.

However, the modernizations of quantum oscillator have been reduced mainly on the solution of some problems considered as technical difficulties, particularly, on the cutting a zero-point energy. At the same time, for the solution of this particular problem there have been required radical modifications of foundations of quantum mechanics. For example, the negative-frequency oscillators to be supposed as cancelling the zero-point energy, the rules relating the wave functions and probabilities have been modified for eliminating the negative norm of states $[1,2]$ (see also [3]), the hypothetical imaginary coordinates, imaginary masses and unreal boundary conditions have been proposed [4]. Nevertheless, all these attempts testified to incompleteness of the conventional quantization procedures and necessity of more careful analysis of their derivation.

In the present paper and in the subsequent papers the results obtained at a sufficiently different approach to this set of problems will be presented. The proposed approach is based on a reasonable conservatism concerning the first principles and replacement of some intuitive hypotheses of the standard quantization to more exact procedures following from the basic symmetries of the systems. At first, we will strongly follow to the restrictions put on the observables by those symmetries, which are characteristic for the relativistic systems, particularly, by the symmetry between particles and antiparticles. Secondly, it is shown that for the solution of the zero-point energy problem in the relativistic systems it is enough to follow their symmetry laws only.

For this reason, the consequences of basic discrete symmetries of particle physics are studied in more general context (see also [5-6]). In the paper a simplest system with the charge conjugation symmetry ( $C$-symmetry) - a system of two harmonic oscillators with opposite signs on frequency - is considered. At representation of this system as a positive-frequency oscillator with a complex generalized coordinate, there appears $U(1)$ symmetry with conserved charge.

In the quantum field theory, at the frequency decomposition of operators of fields, the new creation-annihilation operators $\beta^{*}, \beta$ for positive-frequency antiparticles have been introduced instead of operators of negative-frequency modes. As a result, in the Hamiltonian a zero-point energy had aroused because of "abnormal" product of the such intuitively introduced operators of antiparticles:

$$
H_{0}=\omega\left(a^{*} a+\beta \beta^{*}\right)=\omega\left(a^{*} a+\beta^{*} \beta+1\right) .
$$

In the present paper it is proved that if we define the operators of antiparticles $b^{*}, b$ as a charge-conjugate ones to the operators of particles $a^{*}, a$, the $C$-symmetry requirements for a Hamiltonian and a charge operator lead to the identity:

$$
\beta \beta^{*}=b^{*} b \text {. }
$$


As the result, the zero-point energy in (1) does not arise and the Hamiltonian automatically appears as a normal-ordered and expressed through the mutually charge-conjugate operators:

$$
H_{0}=\omega\left(a^{*} a+b^{*} b\right)
$$

In the case of an oscillator with a complex generalized coordinate the negative-frequency quanta of the same charge evolving backward in time appear as the charge-conjugate quanta of the positive frequency ones evolving forward in time. As a result, the symmetry under the changing a sign of frequency turns to the charge conjugation symmetry. Thus, the ground state becomes charge-conjugation symmetric and its mean energy and charge vanish.

The standard uncertainty relations to which refer at proving inevitability of zero-point fluctuations of the harmonic oscillator and relativistic fields, are valid only for hermitian canonical variables. In the paper a generalization of the uncertainty relations for non-hermitian canonical variables is derived and it is shown that they look like:

$$
|\delta q|^{2}|\delta p|^{2} \geq \frac{\hbar^{2}}{4}\langle Q\rangle^{2}
$$

where $\langle Q\rangle$ - a mean value of the charge operator $i\left(q^{*} p^{*}-p q\right)$ in the given state. As in the charge-conjugation symmetric systems, for example, in case of relativistic fields, the ground state charge vanishes $\langle Q\rangle_{0}=0$ and the unperturbed ground state of these systems represents unquantized external field which, obviously, has not a zero-point energy.

In the first section of the paper the canonical formalism for the complex harmonic oscillator is constructed, as it is accepted in the theory of fields. However, there new operators $\beta^{*}, \beta$, introduced at frequency decomposition of generalized coordinates, do not interpreted at once as ladder operators for the charge-conjugate states. In section 2 an operator identity between the bilinear products $\beta \beta^{*}=b^{*} b$ is obtained using the $C$-symmetry requirements and it is shown that the zero-point charge and the zero-point energy do not arise. In section 3 the new uncertainty relations for non-hermitian canonical variables are derived. In section 4 it is shown that the $C$-symmetric interactions do not change this operator identity. In sections 5 and 6 interactions with a gauge field and with the anharmonic potential are considered.

\section{Canonical formalism for the complex harmonic oscillator}

The system of two harmonic oscillators with real valued coordinates $q_{1}, q_{2}$, unit masses and opposite signs on frequency $\omega_{1}=-\omega_{2}=\omega$ can be presented as a positive-frequency oscillator with the complex generalized coordinates:

$$
q=\left(q_{1}+i q_{2}\right) / \sqrt{2}, \quad q^{*}=\left(q_{1}-i q_{2}\right) / \sqrt{2}
$$

and the Lagrangian: 


$$
L=\left(\partial_{t} q^{*}\right)\left(\partial_{t} q\right)-\omega^{2} q^{*} q, \quad\left(\partial_{t} \equiv \partial / \partial t\right)
$$

Canonical momenta and the Hamiltonian of the system look like:

$$
\begin{gathered}
p=\frac{\partial L}{\partial\left(\partial_{t} q\right)}=\partial_{t} q^{*}, \quad p^{*}=\frac{\partial L}{\partial\left(\partial_{t} q^{*}\right)}=\partial_{t} q, \\
H=p\left(\partial_{t} q\right)+\left(\partial_{t} q^{*}\right) p^{*}-L=p p^{*}+\omega^{2} q^{*} q .
\end{gathered}
$$

Equations of motion for the complex oscillator are:

$$
\partial_{t}^{2} q+\omega^{2} q^{2}=0, \quad \partial_{t}^{2} q^{*}+\omega^{2} q^{* 2}=0,
$$

and quantization leads to the commutators:

$$
i[p, q]=1, \quad i\left[p^{*}, q^{*}\right]=1 \text {. }
$$

A general solution of the equations of motion contains modes both with positive and negative frequencies. In the present paper initially we will exclude negative-frequency states, as it is accepted in quantum field theory, by introducing instead the annihilation operator $a_{-\omega}$ of the negative-frequency quanta a creation operator $\beta^{*}$ of positive-frequency quanta. It means that the system of two oscillators with opposite signs on frequency is represented as a system of two types of positivefrequency oscillators with opposite charges.

Then the decomposition of generalized coordinates and momenta on the frequency components looks like:

$$
\begin{array}{ll}
q=\frac{1}{\sqrt{2 \omega}}\left(a e^{-i \omega t}+\beta^{*} e^{i \omega t}\right), & q^{*}=\frac{1}{\sqrt{2 \omega}}\left(a^{*} e^{i \omega t}+\beta e^{-i \omega t}\right), \\
p=\frac{i \omega}{\sqrt{2 \omega}}\left(a^{*} e^{i \omega t}-\beta e^{-i \omega t}\right), & p^{*}=\frac{-i \omega}{\sqrt{2 \omega}}\left(a e^{-i \omega t}-\beta^{*} e^{i \omega t}\right) .
\end{array}
$$

Since the generalized coordinates $q^{*}, q$ and momenta $p^{*}, p$ are non-hermitian, the ladder operators $a^{*}, a$ commutate with $\beta^{*}, \beta$.

The inversed formulas look like:

$$
\begin{array}{ll}
a=\frac{e^{i \omega t}}{\sqrt{2 \omega}}\left(\omega q+i p^{*}\right), & a^{*}=\frac{e^{-i \omega t}}{\sqrt{2 \omega}}\left(\omega q^{*}-i p\right), \\
\beta=\frac{e^{i \omega t}}{\sqrt{2 \omega}}\left(\omega q^{*}+i p\right), & \beta^{*}=\frac{e^{-i \omega t}}{\sqrt{2 \omega}}\left(\omega q-i p^{*}\right) .
\end{array}
$$

Corresponding non-zero commutators for two kinds of ladder operators, following from (10), are:

$$
\left[a, a^{*}\right]=1, \quad\left[\beta, \beta^{*}\right]=1 .
$$

The Hamiltonian (8) expressed through these operators is:

$$
H=\omega\left(a^{*} a+\beta \beta^{*}\right) \text {. }
$$

Here the product of operators $\beta \beta^{*}$ is "abnormally" ordered. 
The standard approach to quantization of complex fields (represented as sets of complex oscillators) had based on the assumption that the operators $\beta^{*}, \beta$ are charge-conjugate to the ladder operators $a^{*}, a$. As a result of this hypothesis, after the obvious replacement of $\beta \beta^{*}$ to $\beta^{*} \beta+1$, there aroused a zero-point energy and a zero-point charge. However, as it will be shown further in section 3 , the such standard intuitive identification of the operators $\beta^{*}, \beta$ in fact appears incorrect, since leads to the $C$-symmetry violation.

\section{Symmetries of complex oscillator and their consequences}

In the case of an oscillator with complex generalized coordinates and the Lagrangian (6) a global $U(1)$ symmetry takes place:

$$
q^{\prime}=U q U^{-1}, \quad U=\exp (i \theta Q)
$$

with conserved charge $\partial_{t} Q=0$, where the charge operator $Q$ is defined as:

$$
\begin{gathered}
\frac{\delta L}{\delta \theta}=\frac{\partial L}{\partial\left(\partial_{t} q\right)}(-i q)+\left(i q^{*}\right) \frac{\partial L}{\partial\left(\partial_{t} q^{*}\right)}=Q, \\
Q=i\left[q^{*} \partial_{t} q-\left(\partial_{t} q^{*}\right) q\right]=i\left(q^{*} p^{*}-p q\right)=a^{*} a-\beta \beta^{*} .
\end{gathered}
$$

This charge operator contains products of generalized coordinates and momenta and at quantization there is an ordering problem. In a generalization of the Nöther theorem for complex coordinates (17) it is convenient if the second term is hermite-conjugate to the first term, that leads to the obviously hermitian charge operator (18). Further in this paper it will be considered only the charge operator (18) following from the standard Lagrangian (6), while more complicated orderings will be considered in subsequent publications.

In the system of two oscillators with the same masses and frequencies $\omega_{1}=\omega_{2}=\omega$ quanta of two oscillators are indistinguishable and there is a symmetry under their interchanging.

If oscillators differ with the frequency signs $\omega_{1}=-\omega_{2}=\omega$ at interchanging of quanta of two types it is necessary to change and signs of their frequencies also. When this system is represented as the positive-frequency complex oscillator containing two types of quanta of opposite charge, the former symmetry is transformed to the charge conjugation symmetry ( $C$-symmetry). The charge conjugation $C$ is defined conventionally as operation at which quanta with a certain charge are replaced to quanta of an opposite charge. Thus, the Hamiltonian is invariant and the charge operator changes a sign:

$$
\begin{aligned}
& H_{c} \equiv C H C^{-1}=H, \\
& Q_{c} \equiv C Q C^{-1}=-Q .
\end{aligned}
$$

The presence of this symmetry operation leads to the existence of the ladder operators charge-conjugate to earlier introduced ones: 


$$
\begin{aligned}
& b=C a C^{-1}, \quad b^{*}=C a^{*} C^{-1}, \\
& \alpha=C \beta C^{-1}, \quad \alpha^{*}=C \beta^{*} C^{-1},
\end{aligned}
$$

with commutators following from (14):

$$
i\left[b, b^{*}\right]=1, \quad i\left[\alpha, \alpha^{*}\right]=1 .
$$

Operators $a, a^{*}$ and $b, b^{*}$ define the ground and excited states $\left|n_{ \pm}\right\rangle$for the opposite charge quanta:

$$
\begin{array}{ll}
a\left|0_{+}\right\rangle=0, & a\left|n_{+}\right\rangle=\sqrt{n_{+}}\left|n_{+}-1\right\rangle, \quad a^{*}\left|n_{+}\right\rangle=\sqrt{n_{+}+1}\left|n_{+}+1\right\rangle, \\
b\left|0_{-}\right\rangle=0, & b\left|n_{-}\right\rangle=\sqrt{n_{-}}\left|n_{-}-1\right\rangle, \quad b^{*}\left|n_{-}\right\rangle=\sqrt{n_{-}+1}\left|n_{-}+1\right\rangle .
\end{array}
$$

In particular, in the states the charge conjugation rearranges the occupation numbers of quanta with positive and negative charges:

$$
C\left|n_{1+}, n_{2-}\right\rangle=\left|n_{2+}, n_{1-}\right\rangle \text {. }
$$

The corresponding charge-conjugate generalized coordinates and momenta:

$$
\begin{array}{ll}
q_{c}=\frac{1}{\sqrt{2 \omega}}\left(b e^{-i \omega t}+\alpha^{*} e^{i \omega t}\right), & q_{c}^{*}=\frac{1}{\sqrt{2 \omega}}\left(b^{*} e^{i \omega t}+\alpha e^{-i \omega t}\right), \\
p_{c}=\frac{i \omega}{\sqrt{2 \omega}}\left(b^{*} e^{i \omega t}-\alpha e^{-i \omega t}\right), & p_{c}^{*}=\frac{-i \omega}{\sqrt{2 \omega}}\left(b e^{-i \omega t}-\alpha^{*} e^{i \omega t}\right) .
\end{array}
$$

satisfy the commutators:

$$
i\left[p_{c}, q_{c}\right]=1, \quad i\left[p_{c}^{*}, q_{c}^{*}\right]=1 .
$$

Inverse formulas for new ladder operators look like:

$$
\begin{array}{ll}
b=\frac{e^{i \omega t}}{\sqrt{2 \omega}}\left(\omega q_{c}+i p_{c}^{*}\right), & b^{*}=\frac{e^{-i \omega t}}{\sqrt{2 \omega}}\left(\omega q_{c}^{*}-i p_{c}\right), \\
\alpha=\frac{e^{i \omega t}}{\sqrt{2 \omega}}\left(\omega q_{c}^{*}+i p_{c}\right), & \alpha^{*}=\frac{e^{-i \omega t}}{\sqrt{2 \omega}}\left(\omega q_{c}-i p_{c}^{*}\right) .
\end{array}
$$

Wave functions of a ground state for quanta of both types:

$$
\left|0_{+}\right\rangle=\psi_{0+}\left(q^{*}, q\right)=\sqrt{\frac{2 \omega}{\pi}} e^{-\omega q^{*} q}, \quad\left|0_{-}\right\rangle=\psi_{0-}\left(q_{c}^{*}, q_{c}\right)=\sqrt{\frac{2 \omega}{\pi}} e^{-\omega q_{c}^{*} q_{c}}
$$

normalized as:

$$
\int d q^{*} d q \psi_{0+}^{2}\left(q^{*}, q\right)=\int d q_{c}^{*} d q_{c} \psi_{0-}^{2}\left(q_{c}^{*}, q_{c}\right)=1 .
$$

The charge-conjugate Hamiltonian $H_{c}$ and the charge operator $Q_{c}$ thus express through new operators introduced in (26) as:

$$
\begin{aligned}
& H_{c}=\omega\left(b^{*} b+\alpha \alpha^{*}\right), \\
& Q_{c}=b^{*} b-\alpha \alpha^{*} .
\end{aligned}
$$

If $C$-symmetry requirements (19) to write in the expanded form: 


$$
\begin{aligned}
& H=\omega\left(a^{*} a+\beta \beta^{*}\right)=\omega\left(b^{*} b+\alpha \alpha^{*}\right)=H_{c}, \\
& Q=a^{*} a-\beta \beta^{*}=-\left(b^{*} b-\alpha \alpha^{*}\right)=-Q_{c},
\end{aligned}
$$

then we obtain two constraints for four bilinear combinations of ladder operators:

$$
\begin{aligned}
& a^{*} a+\beta \beta^{*}=b^{*} b+\alpha \alpha^{*}, \\
& a^{*} a-\beta \beta^{*}=-b^{*} b+\alpha \alpha^{*} .
\end{aligned}
$$

By adding and subtracting these equalities, two products of auxiliary operators $\beta \beta^{*}$ and $\alpha \alpha^{*}$ we can express through products of operators of mutually chargeconjugate quanta $a^{*} a$ and $b^{*} b$ :

$$
\begin{aligned}
& \alpha \alpha^{*}=a^{*} a, \\
& \beta \beta^{*}=b^{*} b .
\end{aligned}
$$

These relations allow us to calculate the most of matrix elements of products of auxiliary operators.

Thus, using the found identities (32), the expressions for the Hamiltonian and the charge now we can write in the final form:

$$
\begin{aligned}
& H=\omega\left(a^{*} a+b^{*} b\right)=H_{c}, \\
& Q=a^{*} a-b^{*} b=-Q_{c} .
\end{aligned}
$$

Here a non-trivial character of the situation consists in that the operators $b, b^{*}$, defining the states and observables for charge-conjugate quanta, enter into the decomposition of generalized coordinates and momenta (11) not directly, but through the auxiliary operators $\beta^{*}, \beta$, which are related by them only through the bilinear combinations (32).

If, as it is conventionally accepted in quantum field theory, we initially would identify operators $\beta, \beta^{*}$ with $b, b^{*}$ by assuming that $\beta, \beta^{*}$ are charge-conjugate to $a, a^{*}$, we would come to C-symmetry violation. Really, the standard intuitive hypothesis:

$$
\beta=b, \beta^{*}=b^{*}
$$

means, according (32), that $\beta \beta^{*}=\beta^{*} \beta$ and $b b^{*}=b^{*} b$ and further, after the charge conjugation, $a a^{*}=a^{*} a$. Since the ladder operators $a, a^{*}$ cannot commutate, for preserving the hypothesis (34), the C-symmetry requirements (19) should be broken.

We have done opposite and, by preserving the symmetry requirements (19), have not introduce the hypothesis (34). Thus, requirements (19) then have allowed to find the constraints (32) between these two sets of ladder operators and to express $\beta \beta^{*}$ through $b^{*} b$. 


\section{New uncertainty relations for complex canonical variables and a ground state energy}

Harmonic oscillator had played a key role at all stages of creation and application of quantum theory. History almost each new physical property of quantum systems began with application to this simple problem. One of them is the uncertainty relations, the application of which to fluctuations of coordinates and momenta of an oscillating particle in a ground state of the harmonic oscillator with a simple deriving of its zero-point energy was a triumph of this theory.

Therefore, the attempts of quantization of systems without a zero-point energy intuitively have been considered as attempts to exit beyond the standard quantum mechanics. However, it is also well known that many systems with various potentials and symmetries have not a zero-point energy and their ground state energy vanishes without contradiction with quantum mechanics.

In the present paper the negative-frequency quanta are presented as the charge-conjugate quanta with positive energy. As shown in the previous section, it appears that the effect of vanishing of a zero-point energy here takes place also and there appears a question about the uncertainty relations for the such ground state. Thus, we consider this problem for the such systems.

The standard derivation of the Heisenberg uncertainty relations is valid only for hermitian canonical variables only. In our case, where the canonical variables are non-hermitian, corresponding generalization of the uncertainty relations is required.

Definitions of mean square fluctuations, when mean values are equal to zero, look like:

$$
\begin{gathered}
|\delta q|^{2}=\overline{q^{*} q}=\int d q^{*} d q \psi^{*}|q|^{2} \psi, \\
|\delta p|^{2}=\overline{p^{*} p}=\int d q^{*} d q \psi^{*}|p|^{2} \psi=-\hbar^{2} \int d q^{*} d q \psi^{*} \frac{\partial}{\partial q} \frac{\partial}{\partial q^{*}} \psi .
\end{gathered}
$$

Here the momentum operators are defined as:

$$
p \psi=-i \hbar \frac{\partial}{\partial q} \psi, \quad p^{*} \psi=-i \hbar \frac{\partial}{\partial q^{*}} \psi .
$$

Let's consider generalization of a standard inequality with the positive-defined quadratic form for the complex canonical variables:

$$
\int d q^{*} d q\left(\lambda q^{*} \psi^{*}+\frac{\partial \psi^{*}}{\partial q}\right)\left(\lambda q \psi+\frac{\partial \psi}{\partial q^{*}}\right) \geq 0,
$$

where $\lambda$ - a real number. Here, except terms, proportional $|\delta q|^{2}$ and $|\delta p|^{2}$, there are also cross terms:

$$
\begin{aligned}
& \int d q^{*} d q\left(\psi_{n}^{*} q^{*} \frac{\partial \psi_{n}}{\partial q^{*}}+\frac{\partial \psi_{n}^{*}}{\partial q} q \psi_{n}\right)= \\
& =\frac{i}{\hbar} \int d q^{*} d q \psi_{n}^{*}\left(q^{*} p^{*}-p q\right) \psi_{n}=\langle n|Q| n\rangle=\langle Q\rangle,
\end{aligned}
$$


where $Q$ is the charge operator (18).

For the harmonic oscillator with real valued canonical variables the expression $q^{*} p^{*}-p q$ turns to the commutator $(q p-p q)=i \hbar$, the matrix element to $\langle n|Q| n\rangle=-1$, which then leads to the standard uncertainty relation:

$$
\delta q^{2} \delta p^{2} \geq \frac{\hbar^{2}}{4}
$$

In above considering more general case the inequality (38) turns to a quadratic inequality for $\lambda$ :

$$
|\delta q|^{2} \lambda^{2}+\lambda\langle Q\rangle+\frac{1}{\hbar^{2}}|\delta p|^{2} \geq 0
$$

This inequality has real solutions at negative discriminant from which follows:

$$
|\delta q|^{2}|\delta p|^{2} \geq \frac{\hbar^{2}}{4}\langle Q\rangle^{2}
$$

This is the new uncertainty relations for the non-hermitian canonical variables.

Further, since the ground state charge is equal to zero because of chargeconjugation symmetry:

$$
\langle 0|Q| 0\rangle=0,
$$

the inequalities (41) and (42) become trivial and such as in the classical mechanics:

$$
\begin{gathered}
|\delta q|^{2} \lambda^{2}+\frac{1}{\hbar^{2}}|\delta p|^{2} \geq 0, \\
|\delta q|^{2}|\delta p|^{2} \geq 0 .
\end{gathered}
$$

This means that in the ground state of the unperturbed complex oscillator where there are no quanta, the canonical variables are not quantized and a state is the same, as in classical mechanics.

It is clear that for the one-particle states of the nonrelativistic quantum mechanics it is impossible. But this is the case for most of relativistic fields, the canonical variables of which are non-hermitian and the observables are chargeconjugation symmetric. In this case the generalized uncertainty relations (42), at vanishing of the ground state charge, lead to (45) and testify that the ground state of relativistic fields is unquantized external field which does not fluctuate. Thus, the vacuum energy density of such external field, as well as in classical case, has not a zero-point energy.

\section{Conservation of the operator identities for $\boldsymbol{C}$-symmetric interactions}

Let us consider further the $C$-symmetric potentials since, at first, the new properties of a complex oscillator mainly follow from this symmetry and, secondly, in the most important applications of the complex oscillator real interactions possess this symmetry (at least, in a combination with other symmetries).

Thus, in addition to the symmetry requirements for the unperturbed case (19) we have now the $C$-symmetry condition for a potential also: 


$$
V\left(q^{*}, q\right)=V_{c}\left(q_{c}^{*}, q_{c}\right)
$$

where $V_{c}=C V C^{-1}$. The full Hamiltonian also will be $C$-symmetric and, for the potentials, not changing the charge operator, will satisfy similar requirements:

$$
\begin{aligned}
& H=\omega\left(a^{*} a+\beta \beta^{*}\right)+V=\omega\left(b^{*} b+\alpha \alpha^{*}\right)+V_{c}=H_{c}, \\
& Q=a^{*} a-\beta \beta^{*}=-\left(b^{*} b-\alpha \alpha^{*}\right)=-Q_{c} .
\end{aligned}
$$

As we see, thanks to the $C$-symmetry of the potential (46), in the requirement for the Hamiltonian (47) contributions of the interaction potentials in both sides of the equality cancel. As a result, the requirement (47) for the full Hamiltonian is reduced to the former requirement (19) for the unperturbed Hamiltonian, which means the validity of former operator identities (32) in the presence of the $C$-symmetric interaction potentials also.

If a contribution of the interaction potential is calculated in perturbation theory, there are $C$-symmetry requirements for matrix elements in each order of perturbation theory. Energies of the charge-conjugate levels thus should be identical and the matrix elements are related by the set of requirements:

$$
\begin{aligned}
& \left\langle n_{+}|V| n_{+}\right\rangle=\left\langle n_{+}\left|C^{-1} V_{c} C\right| n_{+}\right\rangle=\left\langle n_{-}\left|V_{c}\right| n_{-}\right\rangle, \\
& \left\langle n_{+}|V| n_{ \pm}{ }^{\prime}\right\rangle\left\langle n_{ \pm}{ }^{\prime}|V| n_{+}\right\rangle=\left\langle n_{-}\left|V_{c}\right| n_{\mp}{ }^{\prime}\right\rangle\left\langle n_{\mp}{ }^{\prime}\left|V_{c}\right| n_{-}\right\rangle, \ldots
\end{aligned}
$$

Let's consider $U(1)$ symmetry with a time-dependent parameter $\theta(t)$ :

$$
q^{\prime}=U q U^{-1}, \quad U=\exp [i \theta(t) Q],
$$

when interaction with an external gauge field $\phi$ is introduced by a covariant time derivatives $D_{t}=\partial_{t}-i \phi$. The field transforms as $\phi^{\prime}=\phi+i \partial_{t} \theta$ and at a charge conjugation changes a sign: $\phi_{c}=C \phi C^{-1}=-\phi$. Including of this interaction into the Lagrangian gives:

$$
L=\left(D_{t} q\right)^{*}\left(D_{t} q\right)-\omega^{2} q^{*} q=L_{0}+Q_{\phi} \phi,
$$

where $L_{0}$ is the unperturbed Lagrangian. Then the full Hamiltonian and the charge operator with an external field look like:

$$
\begin{aligned}
& H=H_{0}+\phi Q+\phi^{2} q^{*} q \\
& Q_{\phi}=Q+\phi q^{*} q .
\end{aligned}
$$

Here the $C$-symmetry requirements for them are:

$$
H=H_{c}, \quad Q_{\phi}=-Q_{\phi c} .
$$

which in more detailed form look like:

$$
\begin{aligned}
& H_{0}+\phi Q+\phi^{2} q^{*} q=H_{0 c}-\phi Q_{c}+\phi^{2} q_{c}^{*} q_{c}, \\
& Q+\phi q^{*} q=-\left(Q_{c}-\phi q_{c}^{*} q_{c}\right) .
\end{aligned}
$$


Using the former symmetry requirements for the unperturbed Hamiltonian and the charge, we obtain a new constraint:

$$
q^{*} q=q_{c}^{*} q_{c}
$$

which, after substitution of explicit expressions, gives:

$$
e^{2 i \omega t}\left(a^{*} \beta^{*}-b^{*} \alpha^{*}\right)=e^{-2 i \omega t}(\alpha b-\beta a) \text {. }
$$

Then, particularly, we obtain relations between matrix elements:

$$
\begin{aligned}
& e^{2 i \omega t}\left\langle 1,1\left|\left(a^{*} \beta^{*}-b^{*} \alpha^{*}\right)\right| 0,0\right\rangle=e^{-2 i \omega t}\langle 1,1|(\alpha b-\beta a)| 0,0\rangle=0, \\
& e^{-2 i \omega t}\langle 0,0|(\alpha b-\beta a)| 1,1\rangle=e^{2 i \omega t}\left\langle 0,0\left|\left(a^{*} \beta^{*}-b^{*} \alpha^{*}\right)\right| 1,1\right\rangle=0,
\end{aligned}
$$

which give additional relations for matrix elements of auxiliary operators:

$$
\begin{aligned}
& \left\langle 0,1\left|\beta^{*}\right| 0,0\right\rangle=\left\langle 1,0\left|\alpha^{*}\right| 0,0\right\rangle, \\
& \langle 0,0|\beta| 0,1\rangle=\langle 0,0|\alpha| 1,0\rangle .
\end{aligned}
$$

Thus, the identities for the ladder operators (32) do not change for $C$ symmetric interactions and the symmetry gives the additional identities (57).

\section{A ground state energy at perturbation by the gauge field}

It is important to study how a ground state energy varies at including of interaction, when there is no a zero-point energy in the unperturbed Hamiltonian. We will consider at first perturbation by an external gauge field.

Since the charge operator $Q$ is diagonal and the ladder operators are normalordered, $Q$ does not contribute to the vacuum expectations in all orders of perturbation theory.

Nonzero vacuum expectations can be only from the term $\phi q^{*} q$ with the external field. However, at first, these contributions evidently disappear at removal of the external field and secondly, it is possible to show that at $C$-symmetry they disappear at a nonzero external field also.

Really, in the first order of perturbation theory we have:

$$
\begin{aligned}
& 2 \omega\langle 0|H| 0\rangle=2 \omega \phi^{2}\left\langle 0\left|q^{*} q\right| 0\right\rangle= \\
& =\left\langle 0\left|\left(a^{*} a+\beta \beta^{*}+e^{2 i \omega t} a^{*} \beta^{*}+e^{-2 i \omega t} \beta a\right)\right| 0\right\rangle= \\
& =\phi^{2}\left\langle 0\left|\beta \beta^{*}\right| 0\right\rangle=\phi^{2}\left\langle 0\left|b^{*} b\right| 0\right\rangle=0 .
\end{aligned}
$$

Due to the coordinates commutate, the similar result will be obtained at other orderings and for $\mathrm{C}$-conjugate case also:

$$
\begin{aligned}
& 2 \omega\left\langle 0\left|q q^{*}\right| 0\right\rangle=\left\langle 0\left|a a^{*}+\beta^{*} \beta\right| 0\right\rangle=\left\langle 0\left|a^{*} a+\beta \beta^{*}\right| 0\right\rangle=0 . \\
& 2 \omega\left\langle 0\left|q_{c}^{*} q_{c}\right| 0\right\rangle=\left\langle 0\left|b^{*} b+\alpha \alpha^{*}\right| 0\right\rangle=\left\langle 0\left|b^{*} b+a^{*} a\right| 0\right\rangle=0 .
\end{aligned}
$$

Matrix elements from $\left(q^{*} q\right)^{2}$ and $\left(q_{c}^{*} q_{c}\right)^{2}$, appearing in the second order of perturbation theory, taken without time factors, also vanish due to the operator identities: 


$$
\sum_{n_{1}, n_{2}}\left\langle 0\left|q^{*} q\right| n_{1}, n_{2}\right\rangle\left\langle n_{1}, n_{2}\left|q^{*} q\right| 0\right\rangle \sim\langle 0|\beta| 0,1\rangle\left\langle 0,1\left|\beta^{*}\right| 0\right\rangle=\left\langle 0\left|\beta \beta^{*}\right| 0\right\rangle=0 .
$$

Since the coordinates commutate, all these results also do not depend on ordering. A differently vanish only matrix elements from cross terms for which it is necessary to use the relations (57):

$$
\begin{aligned}
& \sum_{n_{1}, n_{2}}\left\langle 0\left|q^{*} q\right| n_{1}, n_{2}\right\rangle\left\langle n_{1}, n_{2}\left|q_{c}^{*} q_{c}\right| 0\right\rangle \sim\langle 0,0|\beta| 0,1\rangle\left\langle 1,0\left|\alpha^{*}\right| 0,0\right\rangle= \\
& =\langle 0,0|\beta| 0,1\rangle\left\langle 0,1\left|\beta^{*}\right| 0,0\right\rangle=\left\langle 0,0\left|\beta \beta^{*}\right| 0,0\right\rangle=0 .
\end{aligned}
$$

Lack of contributions to the ground state energy of higher orders of perturbation theory can be shown by the similar way. The fact is that all matrix elements of the higher orders contain two gauge-invariant elementary matrix elements $\left\langle 0\left|q^{*} q\right| n_{1}, n_{2}\right\rangle$ and $\left\langle n_{1}, n_{2}\left|q^{*} q\right| 0\right\rangle$ the product of which disappears:

$$
\begin{aligned}
& \sum_{\substack{n_{1}, n_{2}, n_{1}, n_{2}, \ldots}}\left\langle 0\left|q^{*} q\right| n_{1}, n_{2}\right\rangle\left\langle n_{1}, n_{2}\left|q^{*} q\right| n_{1}{ }^{\prime}, n_{2}{ }^{\prime}\right\rangle \ldots\left\langle n_{1}{ }^{\prime}, n_{2}{ }^{\prime}\left|q^{*} q\right| 0\right\rangle= \\
& \quad=\sum_{n_{1}, n_{2}}\left\langle 0\left|q^{*} q\right| n_{1}, n_{2}\right\rangle\left\langle n_{1}, n_{2}\left|q^{*} q\right| 0\right\rangle \sum_{n_{1}, n_{2}{ }^{\prime} \ldots}\left\langle n_{1}, n_{2}\left|q^{*} q\right| n_{1}{ }^{\prime}, n_{2}{ }^{\prime}\right\rangle \ldots=0 .
\end{aligned}
$$

The effects related to time-dependent and non-uniform perturbations and also induced creation of pairs of quanta in the presence of other quanta in case of a complex oscillator will be considered in forthcoming publications.

\section{The ground state energy at anharmonic perturbations}

The symmetries of a complex oscillator admit only gauge-invariant potentials, containing the terms $\left(q^{*} q\right)^{n}$ and $\left(q_{c}^{*} q_{c}\right)^{n}$ (including their products), conserving a charge. From the admissible potentials we have already considered $n=1$ (the harmonic potential) and further we will consider anharmonic 4-order potential $(n=2)$ obeying the $C$-symmetry

$$
V=\lambda\left(q^{*} q+q_{c}^{*} q_{c}\right)^{2}
$$

where $\lambda$ is a dimensional constant of interaction.

The contribution to the ground state energy in the first order of perturbation theory disappears due to the operator identities:

$$
\begin{gathered}
\left\langle 0\left|V_{(a)}^{(1)}\right| 0\right\rangle=\lambda\left\langle 0\left|q^{*} q^{*} q q\right| 0\right\rangle=\lambda\left\langle 0\left|a^{*} a^{*} \beta \beta+\beta \beta \beta^{*} \beta^{*}\right| 0\right\rangle=0 . \\
\left\langle 0\left|V_{(a)}^{c(1)}\right| 0\right\rangle=\lambda\left\langle 0\left|q_{c}^{*} q_{c}^{*} q_{c} q_{c}\right| 0\right\rangle=\lambda\left\langle 0\left|b^{*} b^{*} \alpha \alpha+\alpha \alpha \alpha^{*} \alpha^{*}\right| 0\right\rangle=0 .
\end{gathered}
$$

Other orderings of coordinates will yield the same result. A mixed matrix element also disappears:

$$
\left\langle 0\left|V_{(m)}^{(1)}\right| 0\right\rangle=\lambda\left\langle 0\left|q^{*} q_{c}^{*} q q_{c}\right| 0\right\rangle=\left\langle 0\left|a^{*} b^{*} \beta^{*} \alpha^{*}+\beta \beta^{*} \alpha \alpha^{*}\right| 0\right\rangle=0
$$

Thus, in the first order of perturbation theory there are no anharmonic contributions to the vacuum energy of the charged scalar field.

Contributions to energy of one-particle states of "bubble" type of diagrams also disappear: 


$$
\begin{aligned}
& \left\langle 1,0\left|V^{(1)}\right| 1,0\right\rangle=\lambda\left\langle 1,0\left|\left(q^{*} q+q_{c}^{*} q_{c}\right)^{2}\right| 1,0\right\rangle=0, \\
& \left\langle 0,1\left|V^{(1)}\right| 0,1\right\rangle=\lambda\left\langle 0,1\left|\left(q^{*} q+q_{c}^{*} q_{c}\right)^{2}\right| 0,1\right\rangle=0 .
\end{aligned}
$$

In the second order the matrix element with transition into the intermediate state with two pairs of quanta and antiquanta, only allowed by the charge conservation, also vanish:

$$
\left\langle 0\left|V^{(2)}\right| 0\right\rangle \sim \lambda^{2}\left\langle 0\left|\left(q^{*} q+q_{c}^{*} q_{c}\right)^{2}\right| 2,2\right\rangle\left\langle 2,2\left|\left(q^{*} q+q_{c}^{*} q_{c}\right)^{2}\right| 0\right\rangle=0 .
$$

Matrix elements in higher orders of perturbation theory also vanish since the initial and final matrix elements, related with the ground state, can be factorized and their product disappears:

$$
\begin{aligned}
& \sum_{\substack{n_{1}, n_{2}, n_{1}, n_{2}, \ldots}}\left\langle 0\left|\left(\phi^{*} \phi\right)^{2}\right| n_{1}, n_{2}\right\rangle\left\langle n_{1}, n_{2}\left|\left(\phi^{*} \phi\right)^{2}\right| n_{1}{ }^{\prime}, n_{2}{ }^{\prime}\right\rangle \ldots\left\langle n_{1}{ }^{\prime \prime}, n_{2}{ }^{\prime}\left|\left(\phi^{*} \phi\right)^{2}\right| 0\right\rangle= \\
= & \sum_{n_{1}, n_{2}}\left\langle 0\left|\left(\phi^{*} \phi\right)^{2}\right| n_{1}, n_{2}\right\rangle\left\langle n_{1}, n_{2}\left|\left(\phi^{*} \phi\right)^{2}\right| 0\right\rangle \sum_{n_{1}, n_{2}{ }^{\prime} \ldots}\left\langle n_{1}, n_{2}\left|\left(\phi^{*} \phi\right)^{2}\right| n_{1}{ }^{\prime}, n_{2}{ }^{\prime}\right\rangle \ldots=0 .
\end{aligned}
$$

Matrix elements for excited states and problems of a renormalization of parameters of the initial Lagrangian will be considered in more details in the forthcoming publications.

\section{Conclusion}

Thus, at introduction of ladder operators of the charge-conjugate quanta the agreement with $C$-symmetry requirements is nontrivial and only new operator identities between the bilinear products of ladder operators allow to take into account correctly the symmetry requirements.

Thus it appears that $C$-symmetry leads to new and also nontrivial effect - to a natural normal ordering of operators and to vanishing of the zero-point energy of the complex oscillator. Moreover, at including of the $C$-symmetric interactions a ground state energy remains equal to zero in all orders of perturbation theory.

For systems with non-hermitian canonical variables a general form of uncertainty relations takes place. From them follows that the ground state of $C$-symmetric systems (for example, relativistic fields) has no zero-point energy and does not fluctuate, remaining as a classical external field.

In forthcoming publications the new method of the $C$-symmetric quantization of the complex harmonic oscillator will be applied to a chain of the coupled oscillators and to relativistic fields.

\section{References}

1. Dirac P.A.M. (1942) Proc. Roy. Soc., London, A114, 243, 710.

2. Pauli W. (1943) Rev. Mod. Phys., 15, 175.

3. Moffat J. (2006) Phys. Lett., B627, 9.

4. 't Hooft G., Nobbenhuis S. (2006) arxiv: 0602076.

5. Zakir Z. (2006) Theor. Phys., Astrophys. and Cosmol., 1, 1, 11; 1, 4, 61; arxiv:0705.0899. 